\title{
Cancer classification: Mutual information, target network and strategies of therapy
}

\author{
Wen-Chin $\mathrm{Hsu}^{1,2}$, Chan-Cheng Liư ${ }^{4}$, Fu Chang ${ }^{4}$ and Su-Shing Chen ${ }^{1,3^{*}}$
}

\begin{abstract}
Background: Cancer therapy is a challenging research area because side effects often occur in chemo and radiation therapy. We intend to study a multi-targets and multi-components design that will provide synergistic results to improve efficiency of cancer therapy.

Methods: We have developed a general methodology, AMFES (Adaptive Multiple FEature Selection), for ranking and selecting important cancer biomarkers based on SVM (Support Vector Machine) classification. In particular, we exemplify this method by three datasets: a prostate cancer (three stages), a breast cancer (four subtypes), and another prostate cancer (normal vs. cancerous). Moreover, we have computed the target networks of these biomarkers as the signatures of the cancers with additional information (mutual information between biomarkers of the network). Then, we proposed a robust framework for synergistic therapy design approach which includes varies existing mechanisms.

Results: These methodologies were applied to three GEO datasets: GSE18655 (three prostate stages), GSE19536 (4 subtypes breast cancers) and GSE21036 (prostate cancer cells and normal cells) shown in. We selected 96 biomarkers for first prostate cancer dataset (three prostate stages), 72 for breast cancer (luminal A vs. luminal B), 68 for breast cancer (basal-like vs. normal-like), and 22 for another prostate cancer (cancerous vs. normal. In addition, we obtained statistically significant results of mutual information, which demonstrate that the dependencies among these biomarkers can be positive or negative.
\end{abstract}

Conclusions: We proposed an efficient feature ranking and selection scheme, AMFES, to select an important subset from a large number of features for any cancer dataset. Thus, we obtained the signatures of these cancers by building their target networks. Finally, we proposed a robust framework of synergistic therapy for cancer patients. Our framework is not only supported by real GEO datasets but also aim to a multi-targets/multi-components drug design tool, which improves the traditional single target/single component analysis methods. This framework builds a computational foundation which can provide a clear classification of cancers and lead to an efficient cancer therapy.

Keywords: Feature selection, Biomarkers, Microarray, Therapy design, Target network

\section{Background}

Cancer therapy is a difficult research area due to its level of complexity. Lately, the mere superposition of single drugs is found to generate side-effects and crosstalk with another drug which may cancel out the final success of treatments. Thus, current research focuses on measuring the drug treatments as a whole rather than considering

\footnotetext{
* Correspondence: suchen@cise.ufl.edu

'System Biology Lab, University of Florida, Florida, USA

${ }^{3}$ Department of Computer and Information Science and Engineering, University of Florida, Florida, USA

Full list of author information is available at the end of the article
}

them individually $[1,2]$. Later, a synergistic concept is proposed to evaluate the drug treatments [3]. However, evaluations are still based on cases and do not have a systematic approach. In [4], a network methodology is first used to evaluate efficiency of drug treatments. Thus, $\mathrm{Li}$ et al. use a parameter, namely a SS (Synergy Score) to introduce the topology factor of the network based on the disease and the drug agent combination [5].

Our approach is first to build a more precise target network from the selected biomarkers (by AMFES) [6]. Then, we identify the intrinsic properties by computing 
mutual information of the interactions among these biomarkers. Our approach is to improve Li's results by considering the mutual information in the target network. And we provide a general framework of synergistic therapy, which may include several different approaches.

\section{Methods AMFES}

The COD (Curse of Dimensionality) has been a major challenge of microarray data analysis due to the large number of genes (features) and relatively small number of samples (patterns). To tackle this problem, many gene selection methodologies were developed to select only significant subsets of genes in a microarray dataset. AMFES selects an optimal subset of genes by training a SVM with subsets of genes generated adaptively [6].

When AMFES runs a dataset, all samples are randomly divided into a training subset $S$ of samples and a testing subset $T$ of samples at a heuristic ratio of 5:1. $S$ is used for ranking and selecting of genes and for constructing a classifier out of the selected genes. $T$ is used for computing test accuracy. When a training subset $S$ is given, we extract $r$ training-validation pairs from $S$ according to the heuristic rule $r=\max$ (5, (int) $(500 / n$ $+0.5)$ ) and $n$ is the number of samples in $S$. Each pair randomly divides $S$ into a training component of samples and a validation component of samples at a ratio of 4:1. The heuristic ratio and rule are chosen based on the experimental experiences at the balance of time consumption and performance. Basically, AMFES has two fundamental processes, ranking and selection. We first explain each process in details and then the integrated version at the end.

\section{Ranking}

The gene ranking process contains a few ranking stages. At first stage, all genes are ranked by their ranking scores in a descending order. Then, in the next stage, only the top half ranked genes are ranked again while the bottom half holds the current order in the subsequent stage. The same iteration repeats recursively until only three genes are remained to be ranked again to complete one ranking process. Assume at a given ranking stage, there are $k$ genes indexed from 1 to $k$. To rank these $k$ genes, we follow 4 steps below. (I) We first generate $m$ independent subsets $S_{1} \ldots S_{m}$. Each subset $S_{i}, i=1,2 \ldots m$, has $j$ genes which are selected randomly and independently from the $k$ genes, where $j=$ (int) $(k / 2)$. (II) Let $C_{i}$ be the SVM classifier that is trained on each subset of genes, $i=1,2 \ldots m$. For each gene of $k$ genes, we compute the ranking score $\theta_{m}$ (g) of the gene $g$, as equation (1). (III) We use the average weight of the gene $g$, the summation of weights of $g$ in $m$ subsets divided by the number of subsets for which $g$ is randomly selected. This increases the robustness to represent the true classifying ability of the gene $g$. (IV) Rank $k$ genes in the descending order by their ranking scores.

$$
\theta_{m}(g)=\frac{\sum_{i=1}^{m} I_{\left\{g \in S_{i}\right\}} \text { weight }_{i}(g)}{\sum_{i=1}^{m} I_{\left\{g \in S_{i}\right\}}}
$$

where $\mathrm{I}$ is an indicator function such that $\mathrm{I}_{\text {proposition }}=1$ if the proposition is true; otherwise, $\mathrm{I}_{\text {proposition }}=0$. In other word, if gene $g$ is randomly selected for the subset $S_{i}$, it is denoted as $g \in S_{i}$ and $\mathrm{I}_{\text {proposition }}=1$.

We denote the objective function of $\mathrm{C}_{i}$ as $o b j_{i}\left(v_{1}, v_{2}, \ldots, v_{s}\right)$ where $\mathbf{v}_{1}, \mathbf{v}_{2} \ldots \mathbf{v}_{\mathbf{s}}$ are support vectors of $\mathrm{C}_{i}$. The weight $_{i}(\mathrm{~g})$ is then defined as the change in the objective function due to g, i.e.,

$$
\text { weight }_{i}(g)=\left|o b j_{i}\left(v_{1}, v_{2}, \ldots v_{s}\right)-o b j_{i}\left(v_{1}^{(g)}, v_{2}^{(g)}, \ldots, v_{3}^{(g)}\right)\right|
$$

[6] $[7,8]$. Note that if $\mathbf{v}$ is a vector, $\mathbf{v}^{(g)}$ is the vector obtained by dropping gene $g$ from $\mathbf{v}$. Let $\theta_{\mathrm{m}}$ be a vector comprising the ranking scores derived from the $m$ gene subsets generated thus far and $\theta_{\mathrm{m}-1}$ is the vector at the previous stage. The $m$ value is determined when $\theta_{\mathrm{m}}$ satisfies the equation (3) by adding a gene to an empty subset once a time.

$$
\frac{\left\|\boldsymbol{\theta}_{m-1}-\boldsymbol{\theta}_{m}\right\|^{2}}{\left\|\boldsymbol{\theta}_{m-1}\right\|^{2}}<0.01
$$

where $\|\theta\|$ is understood as the Euclidean norm of vector $\theta$. The pseudo codes of ranking process are shown in below.

\section{Pseudo codes for ranking process of AMFES}

RANK-SUBROUTINE

INPUT: a subset of $k$ genes to be ranked

Generate $k$ artificial genes and put them next to the original genes

Pick an initial tentative value of $m$

DO WHILE $m$ does not satisfies equation (3)

FOR each subset Si of $m$ subsets

Randomly select $j$ elements from $k$ genes to form the subset Si.

Train an SVM to get weight $i_{i}(g)$ for each gene in the subset

ENDFOR 
FOR each gene of $k$ genes

Compute the average score of the gene from $m$ subsets

\section{ENDFOR}

List $k$ genes in descending order by their ranking scores

\section{ENDDO}

\section{OUPUT: a ranked $k$ genes}

\section{Selection}

Ranking artificial features together with original features has been demonstrated as a useful tool to distinguish relevant features from irrelevant ones as in [9-11]. In our selection process, we also use this technique to find the optimal subset of genes.

Assume a set of genes is given. We generate artificial genes and rank them together with original ones. After finishing ranking the set, we assign a gene-index to each original gene by the proportion of artificial ones that are ranked above it where the gene-index is the real numerical value between 0 and 1 . Then, we generate a few subset candidates from which the optimal subset is chosen. Let $p_{1}, p_{2}$, be the sequence of subset-indices of the candidates with $p_{1}<p_{2}<\ldots$.where $p_{i}=i \times 0.005$ and $i=1,2, \ldots 200$. Let $\mathrm{B}\left(p_{i}\right)$ denote the corresponding subset of subset-index $p_{\dot{v}}$ and it contains original genes whose indices are smaller than or equal to $p_{i}$. Then, we train a SVM on every $\mathrm{B}\left(p_{i}\right)$, and compute its validation accuracy $v\left(p_{i}\right)$.

We stop at the first $p_{k}$ at which $\mathrm{v}\left(p_{k}\right) \geq v_{\text {baseline }}$ and $v$ $\left(p_{k}\right) \geq v\left(p_{l}\right)$ for $k \leq l \leq k+10$, where $v_{\text {baseline }}$ is the validation accuracy rate of the SVM trained on the baseline, i.e., the case in which all features are involved in training. The final result, $\mathrm{B}\left(p_{k}\right)$, is then the optimal subset for the given set of genes. The pseudo codes for selection process of AMFES are listed below.

\section{Pseudo codes for selection process of AMFES}

\section{SELECTION-SUBROUTINE \\ INPUT: a few subsets with their validation accuracies, $a v\left(p_{i}\right)$}

Compute the validation accuracy of all genes, vbaseline. FOR each subset given

IF $v\left(p_{k}\right) \geq v_{\text {baseline }}$ and $v\left(p_{k}\right) \geq v\left(p_{l}\right)$ for $k \leq l \leq k+10$ THEN

Resulted subset is $B\left(p_{k}\right)$

\section{ENDFOR}

\section{OUPUT: $B\left(p_{k}\right)$}

\section{Integrated version}

The ranking and selection processes from previous sections are for one training- validation pair. To increase the reliability of validation, we generate $r$ pairs to find the optimal subset. We calculate the validation accuracy of the $q^{\text {th }}$ pair for all $p_{q-i}$ subsets where $q$ denotes pairindex and $i$ denotes the subset-index. Then, we compute $a v\left(p_{i}\right)$, the average of $v\left(p_{q-i}\right)$ over $r$ training-validation pairs and perform the subset search as explained in selection section on $a v\left(p_{i}\right)$ to find the optimal $p_{i}$, denoted as $p^{*}$.However, $p^{*}$ does not correspond to a unique subset, since each pair has its own $\mathrm{B}\left(p^{*}\right)$ and they can be all different. Thus, we adopt all samples of $S$ as training samples in order to find a unique subset. We generate artificial genes and rank them together with original genes. Finally, we select the original genes whose indices are smaller than or equal to the $p^{*}$ as the genes we select for $S$. The integrated version of process is shown below. In the pseudo codes below, the AMFES-ALGORITHM represents the integrated version of the whole process while RANK-SUBROUTINE represents the ranking process and SELECTION-SUBROUTINE represents the selection process.

\section{Pseudo codes for integrated version of AMFES}

AMFES ALGORITHM-Integrated Version INPUT: a dataset

Divide a dataset into train samples and test samples. Divide the train samples into $r$ training-validation components pairs

FOR each pair of $r$ train-validation components pairs

Generate 200 candidate subsets $p_{q-i}$

FOR each subset of 200 subsets

CALL RANK subroutine to rank each subset. Assign each original gene a gene-index Train each subset on an SVM and compute corresponding validation accuracy, $v\left(p_{q-i}\right)$, for the subset

END FOR

END FOR

FOR each subset of 200 subsets 
Compute average validation rate, av $\left(p_{i}\right)$, of the subset from $r$ pairs.

END FOR

CALL SELECTION subroutine to search for the optimal subset by its average validation rate and denotes it as $p^{*}$

CALL RANK subroutine to rank original genes again and select original genes which belong to the subset $B$ $(p *)$.

OUPUT: an optimal subset of genes B(p*)

\section{Mutual information}

Mutual information has been used to measure the dependency between two random variables based on the probability of them. If two random variables $\mathrm{X}$ and $\mathrm{Y}$, the mutual information of $\mathrm{X}$ and $\mathrm{Y}, \mathrm{I}(\mathrm{X} ; \mathrm{Y})$, can be expressed as these equivalent equations [12]:

$$
\begin{aligned}
& I(X ; Y)=H(X)-H(X \mid Y) \\
& =H(Y)-H(Y \mid X) \\
& =H(X)+H(Y)-H(X, Y)
\end{aligned}
$$

where $H(X), H(Y)$ denote marginal entropies, $H(X \mid Y)$ and $\mathrm{H}(\mathrm{Y} \mid \mathrm{X})$ denote conditional entropies and $\mathrm{H}(\mathrm{X}, \mathrm{Y})$ denotes joint entropy of the $\mathrm{X}$ and $\mathrm{Y}$. To compute entropy, the probability distribution functions of the random variables are required to be calculated first. Because gene expressions are usually continuous numbers, we used the kernel estimation to calculate the probability distribution [13].

Assume the two random variables $\mathrm{X}$ and $\mathrm{Y}$ are continuous numbers. The mutual information is defined as [12]:

$$
I(X, Y)=\iint f(x, y) \log \left(\frac{f(x, y)}{f(x) f(y)}\right) d x d y
$$

where $f(x, y)$ denotes the joint probability distribution, and $f(\mathrm{x})$ and $f(\mathrm{y})$ denote marginal probability distribution of $\mathrm{X}$ and Y. By using the Gaussian kernel estimation, the $f(x, y)$, $f(\mathrm{x})$ and $f(\mathrm{y})$ can be further represented as equations below [14]:

$$
\begin{aligned}
& f(x, y)=\frac{1}{M} \sum_{2 \pi h^{2}} e^{-\frac{1}{2 h^{2}}\left(\left(x-x_{u}\right)^{2}+\left(y-y_{u}^{2}\right)\right)} \\
& f(x)=\frac{1}{M} \sum \frac{1}{\sqrt{2 \pi h^{2}}} e^{-\frac{1}{2 h^{2}}\left(x-y_{u}\right)^{2}},
\end{aligned}
$$

where $M$ represents the number of samples for both $\mathrm{X}$ and Y, $u$ is index of samples $u=1,2, \ldots M$, and $h$ is a parameter controlling the width of the kernels. Thus, the mutual information $I(X, Y)$ can then be represented as:

$$
I(X, Y)=\frac{1}{M} \sum_{i} \log \frac{M \sum_{i} e^{-\frac{1}{2 h^{2}}\left(\left(x_{w}-x_{u}\right)^{2}+\left(y_{w i}-y_{u}\right)^{2}\right)}}{\sum_{j} e^{-\frac{1}{2 h^{2}}\left(x_{w}-x_{u}\right)^{2}} \sum_{j} e^{-\frac{1}{2 h^{2}\left(y_{w i}-y_{u}\right)^{2}}}}
$$

where both $w, u$ are indices of samples $w, u=1,2, \ldots M$.

Computation of pairwise genes of a microarray dataset usually involves nested loops calculation which takes a dramatic amount of time. Assume a dataset has $N$ genes and each gene has $M$ samples. To calculate the pairwise mutual information values, the computation usually first finds the kernel distance between any two samples for a given gene. Then, the same process goes through every pair of genes in the dataset. In order to be computation efficient, two improvements are applied [13]. The first one is to calculate the marginal probability of each gene in advance and use it repeatedly during the process [13] [15].The second improvement is to move the summation of each sample pair for a given gene to the most outer for-loop rather than inside a nested for-loop for every pairwise gene. As a result, the kernel distance between two samples is only calculated twice instead $N$ times which saves a lot of computation time. LNO (Loops Nest Optimization) which changes the order of nested loops is a common time-saving technique in computer science field [16].

\section{Target network}

The effect of drugs with multiple components should be viewed as a whole rather than a superposition of individual components [1] [2]. Thus, a synergic concept is formed and considered as an efficient manner to design a drug [3]. In [17], mathematical models are used to measure the effect generated by the multiple components. However, it does not consider practical situation such as crosstalk between pathways. A network approach starts to be used to analyze the interactions among multiple components [4]. Initiated by work in [4], another system biological methodology, NIMS (Network-target-based Identification of Multicomponent Synergy) is proposed to measure the effect of drug agent pairs depending on their gene expression data [5]. NIMS focuses on ranking the drug agent pairs of Chinese Medicine components by their SS.

In [5], it assumes that a drug component is denoted as a drug agent and with which a set of genes associated are denoted as agent genes of the drug agent. For a given disease, assume there are $N$ drug agents where $N=1,2 \ldots n$. Initially, NIMS randomly chooses two drug agents from $N, \mathrm{~A}_{1}$, and $\mathrm{A}_{2}$, and builds a background target network 
by their agent genes in a graph. From the graph, NIMS calculates TS (Topology Score) of the graph by applying the PCA (Principle Component Analysis) to form a IP value which is integrated by betweenness, closeness and a variant of Eigenvalues PageRank [18]. The TS is used to evaluate the topology significance of the target network for the drug agent pair, $A_{1}$ and $A_{2}$, and is defined as

$$
\begin{aligned}
T S_{1,2}= & \frac{1}{2} \times\left[\frac{\sum_{i} I P_{1}(i) \times \exp \left(-\min \left(d_{i, j}\right)\right)}{\sum_{i} I P_{1}(i)}\right. \\
& \left.+\frac{\sum_{j} I P_{2}(j) \times \exp \left(-\min \left(d_{j, i}\right)\right)}{\sum_{j} I P_{2}(j)}\right],
\end{aligned}
$$

where $I P_{1}$ and $I P_{2}$ denote IP values for drug agent $\mathrm{A}_{1}$ agent and $\mathrm{A}_{2}$. $\operatorname{Min}\left(d_{\mathrm{i}, \mathrm{j}}\right)$ denotes minimum shortest path from gene $i$ of $\mathrm{A}_{1}$ to all genes of $\mathrm{A}_{2}$ and $\min \left(d_{\mathrm{j}, \mathrm{i}}\right)$ denotes the one from gene $j$ of $A_{1}$ to all genes of $A_{2}$.

NIMS define another term, AS (Agent Score), to evaluate the similarity of a disease phenotype for a drug agent. For a given drug agent, if one of its agent genes has a phenotype record in the OMIM (Online Mendelian Inheritance in Man) database, the drug agent has that phenotype as one of its phenotype. The similarity score of a drug agent pair is defined as the cosine value of the pair's feature vector angle [19]. The AS is defined as:

$$
A S_{1,2}=\frac{\sum_{i, j} P_{i, j}}{M},
$$

where $P_{\mathrm{i}, \mathrm{j}}$ denotes similarity score of $i$ th phenotype of $\mathrm{A}_{1}$ and $j$ th phenotype of $\mathrm{A}_{2}$ and $M$ denotes the total number of phenotypes.

The SS of the pair is then defined as the product of TS and AS. NIMS calculates SS for all possible drug agent pairs for a disease and then can find potential drug agent pairs after ranking them by SS.

\section{Results}

\section{MIROARRAY data description}

We made a brief description of these three datasets in Table 1. It listed the number of biomarkers, types of biomarkers, number of samples and variation of samples used.

\section{The prostate cancer dataset with RNA biomarkers}

In order to give a better prognosis, pathologists have used a cancer stage to measure cell tissues and tumors' aggressions as an indicator for doctors to choose a suitable treatment. The most widely used cancer staging system is TNM (Tumor, Node, and Metastasis) system [20]. Depending on levels of differentiation between normal and tumor cells, a different histologic grade is given. Tumors with grade 1 indicate almost normal tissues, with grade 2 indicating somewhat normal tissues and with grade 3 indicating tissues far away from normal conditions. Although most of cancers can be adapted to TNM grading system, some specific cancers require additional grading systems for pathologists to better interpret tumors.

The Gleason Grading System is especially used for prostate cancers and a GS (Gleason Score) is given based on cellular contents and tissues of cancer biopsies from patients. The higher the GS are, the worse the prognoses are. The prostate cancer dataset, GSE18655, includes 139 patients with 502 molecular markers, RNAs [21]. In [21], it showed that prostate tumors with gene fusions, TMPRSS2: ERG T1/E,4 have higher risk of recurrences than tumors without the gene fusions. 139 samples were prostate freshfrozen tumor tissues of patients after a radical prostatectomy surgery. All samples were taken from the patients' prostates at the time of prostatectomy and liquid nitrogen was used to freeze middle sections of prostates at extreme low temperature. Among these patients, 38 patient samples have GS 5-6 corresponding to histologic grade 1, 90 samples have GS 7 corresponding to histologic grade 2 and 11 samples have GS 8-9 corresponding to histologic grade 3. The platform used for the datasets is GPL5858, DASL (cDNA-mediated, annealing, selection, extension and ligation) Human Cancer Panel by Gene manufactured by Illumina. The FDR (false discovery rate) of all RNAs expressions in the microarray is less than $5 \%$.

Table 1 Descriptions of 3 datasets: GSE18655 (prostate cancer), GSE19536 (breast cancer) and GSE21036 (prostate cancer)

\begin{tabular}{llll}
\hline & Prostate Cancer (GSE18655) & Breast Cancer (GSE19536) & Prostate Cancer (GSE21036) \\
\hline Number of Biomarkers & 502 & 489 & 373 \\
\hline Type of Biomarkers & RNAs & miRNAs & miRNAs \\
\hline Number of Samples & 139 & 78 & 142 \\
\hline Variation of Samples & $\begin{array}{l}\text { Grade1(38), Grade2(90), } \\
\text { Grade3(11) }\end{array}$ & $\begin{array}{l}\text { Luminal A ( 41), Luminal B (12), } \\
\text { Basal-like (15), Normal-like(10) }\end{array}$ & Cancerous (114), Normal(28) \\
\hline
\end{tabular}




\section{Breast cancer dataset with Non-coding miRNA biomarkers}

The miRNAs have strong correlation with some cellular processes, such as proliferation, which has been used as a breast cancer dataset [22]. It has 799 miRNAs and 101 patients' samples. Differential expressions of miRNAs indicated different level of proliferations corresponding to 6 intrinsic breast cancer subtypes: luminal A, luminal $\mathrm{B}$, basal-like, normal-like, and ERBB2. The original dataset has 101 samples and among them, 41 samples are luminal A, 15 samples are basal-like, 10 samples are normal-like, 12 samples are luminal B, 17 samples are ERBB2, 2 samples have T35 mutation status, another sample has T35 wide type mutation and 3 samples are not classified. GSE19536 was represented in two platforms GPL8227, an Agilient-09118 Human miRNA microarray 2.0 G4470B (miRNA ID version) and the GPL6480, an Agilent-014850 whole Human Genome Microarray 4x44k G4112F (Probe Name). For this paper, we only used the expressions from GPL8227.

\section{Prostate cancer dataset of cancerous and normal samples with miRNA biomarkers}

The CNAs (Copy Number Alterations) of some genes may associate with growth of prostate cancers [23]. In addition, some changes are discovered in mutations of fusion gene, mRNA expressions and pathways in a majority of primary prostate samples. The analysis was applied to four platforms and consists of 3 subseries, GSE21034, GSE21035 and GSE21036 [23]. For this paper, we only use the GSE 21036 for analysis. The microarray dataset has 142 samples which include 114 primary prostate cancer samples and 28 normal cells samples. The platform is Agilent-019118 Human miRNA Microarray 2.0 G4470B (miRNA ID version).

\section{Results of AMFES}

We employ the AMFES on the prostate cancer (GSE18655), breast cancer (GSE19536) and another prostate cancer (GSE21036) datasets. Consequently, for GSE18655, AMFES selects 96 biomarkers. The classification is performed in two steps. The first step performs classification between grade1 and above samples and it selects 93 biomarkers. At the second step, AMFES classifies between grade 2 and grade 3 samples and it selects 3 biomarkers. Thus, we can assume that these 96 biomarkers can classify among grade1, grade 2 and grade3 samples [6]. For GSE19536, AMFES also performs classification in two steps. At the first step, AMFES classify between luminal and non-luminal types samples and it selects 47 biomarkers [6]. At the second step, AMFES further classifies luminal samples into luminal A and luminal B and selects 27 biomarkers. For the non-luminal samples, AMFES also classifies them into basal-like and normal-like samples and selects 25 biomarkers [6]. After removing duplicate biomarkers, AMFES has 72 (47+27-2(duplicated)) for classifying luminal samples and $68(47+25-4$ (duplicated)) for classifying non-luminal ones [6]. For GSE21036, AMFES simply selects 22 biomarkers for classifying cancerous and normal samples. Table 2. shows the number of selected genes. The complete lists of these biomarkers can be found in Additional file 1 GSE18655_96_Biomarkers.xlsx, Additional file 2 GSE19536_72_Biomarkers.xlsx, Additional file 3 GSE19536_68_Biomarkers.xlsx, and Additional file 4 GSE21036_22_Biomakers.xlsx.

We then apply the MI calculation described in the $\mathrm{Mu}-$ tual Information section on 96 biomarkers for GSE18655 and represent the pairwise MI values of grade 1, grade 2 and grade 3 samples in three 96*96 matrixes which can be found in Additional file 5 GSE18655 Grade1 MI.xlsx, Additional file 6 GSE18655 Grade2 MI.xlsx and Additional file 7 GSE18655 Grade3 MI.xlsx. We also represent the four MI matrixes of 72 and 68 biomarkers for GSE19536 in Additional file 8 GSE19536 Luminal-A MI. xlsx, Additional file 9 GSE19536 Luminal-B MI.xlsx, Additional file 10 GSE19536 Basal-Like MI.xlsx, and Additional file 11 GSE19536 Normal-Like MI.xlsx. The two MI matrixes for GSE21036 are in Additional file 12 GSE21036 Cancer MI.xlsx, Additional file 13 GSE21036 Normal MI.xlsx.

We analyze these MI matrixes and list differences between them under different conditions in Table 3. For a given matrix, the first column in Table 3 denotes the mean value; the second column denotes the standard deviation; the third column shows the number of positive values in the matrix; the fourth column shows the number of negative values; the sixth column shows the minimum value and the seventh column displays the maximum. In the fifth column, we compare MI matrixes under two different conditions such as luminal A vs. luminal B. If the signs of two entries at the same position in these two matrixes are different, we count it as one sign difference. The fifth column denotes the number of sign differences of the samples compared. We employ the same process for comparing basal-like versus

Table 2 Results of selected subsets of genes

\begin{tabular}{lllll}
\hline & $\begin{array}{l}\text { Prostate Cancer } \\
\text { (GSE18655) }\end{array}$ & $\begin{array}{l}\text { Breast Cancer } \\
\text { (GSE19536) }\end{array}$ & $\begin{array}{l}\text { Breast Cancer } \\
\text { (GSE19536) }\end{array}$ & $\begin{array}{l}\text { Prostate Cancer } \\
\text { (GSE21036) }\end{array}$ \\
\hline $\begin{array}{l}\text { Number of Biomarkers } \\
\text { Selected }\end{array}$ & 96 & 72 & 68 & 22 \\
\hline \begin{tabular}{l} 
Variation of Samples \\
\hline
\end{tabular} & Grade1, Grade2, Grade3 & Luminal A, Luminal B & Basal-like Normal-like & Cancerous Normal \\
\hline
\end{tabular}


Table 3 Results of analysis of MI matrices

\begin{tabular}{|c|c|c|c|c|c|c|c|}
\hline & $\begin{array}{l}\text { Mean value } \\
\text { of } \mathrm{MI}\end{array}$ & $\begin{array}{l}\text { Standard } \\
\text { deviation of MI }\end{array}$ & $\begin{array}{l}\text { Num of } \\
\text { positive values }\end{array}$ & $\begin{array}{l}\text { Num of } \\
\text { negative values }\end{array}$ & $\begin{array}{l}\text { Num of values } \\
\text { of different sign }\end{array}$ & $\begin{array}{l}\text { Min } \\
\text { value }\end{array}$ & $\begin{array}{l}\text { Max } \\
\text { value } \\
\end{array}$ \\
\hline GSE18655_grade1 & 0.00024 & 0.0015 & 6298 & 2918 & N/A & -0.0011 & 0.0858 \\
\hline GSE18655_grade2 & 0.00020 & 0.0017 & 6468 & 2748 & & -0.0018 & 0.0949 \\
\hline GSE18655_grade3 & 0.0004 & 0.0021 & 6650 & 2566 & & -0.0029 & 0.0582 \\
\hline GSE19536_A(72) & 0.00036 & 0.0022 & 3912 & 1272 & 2052 & -0.0010 & 0.1293 \\
\hline GSE19536_B(72) & 0.00053 & 0.0040 & 3388 & 1796 & & -0.0022 & 0.2279 \\
\hline GSE19536_BasalLike(68) & 0.0017 & 0.0056 & 3491 & 998 & 1217 & -0.0033 & 0.1648 \\
\hline GSE19536_NormalLike(68) & 0.0056 & 0.008 & 4200 & 420 & & -0.002 & 0.1279 \\
\hline GSE21036_cancer & 0.0165 & 0.0212 & 10 & 474 & 56 & -0.002 & 0.1446 \\
\hline GSE21036_norm & 0.0086 & 0.0146 & 46 & 438 & & -0.0015 & 0.1565 \\
\hline
\end{tabular}

normal-like for GSE19536 and the cancerous versus normal for GSE21036. To visualize the differences, we display the histograms of MI values of grade1s, grade2s and grade3s in Figure 1. Figure 2 shows the histograms for luminal As versus luminal Bs. Figure 3 shows basal-likes versus normal-likes and Figure 4 shows the cancerous versus normals.

For the fifth column of comparison of GSE18655, since there are three types prostate, they cannot be fairly compared, so we skipped the process for it. In addition, because there are many MI entries for all histograms, we only show the densest section of each histogram in figures.

\section{Results of calculating mutual information}

The statistic results of calculating mutual information are shown in Table 3 at the end of this paper.

\section{Synergistic therapy}

Based on the interpretation of the network $[4,5]$, we proposed a framework that can help to elucidate the underlying interactions between multi-target biomarkers and multi-component drug agents. The framework consists of three parts: selecting biomarkers of a complex disease such as cancer, building target networks of biomarkers, and

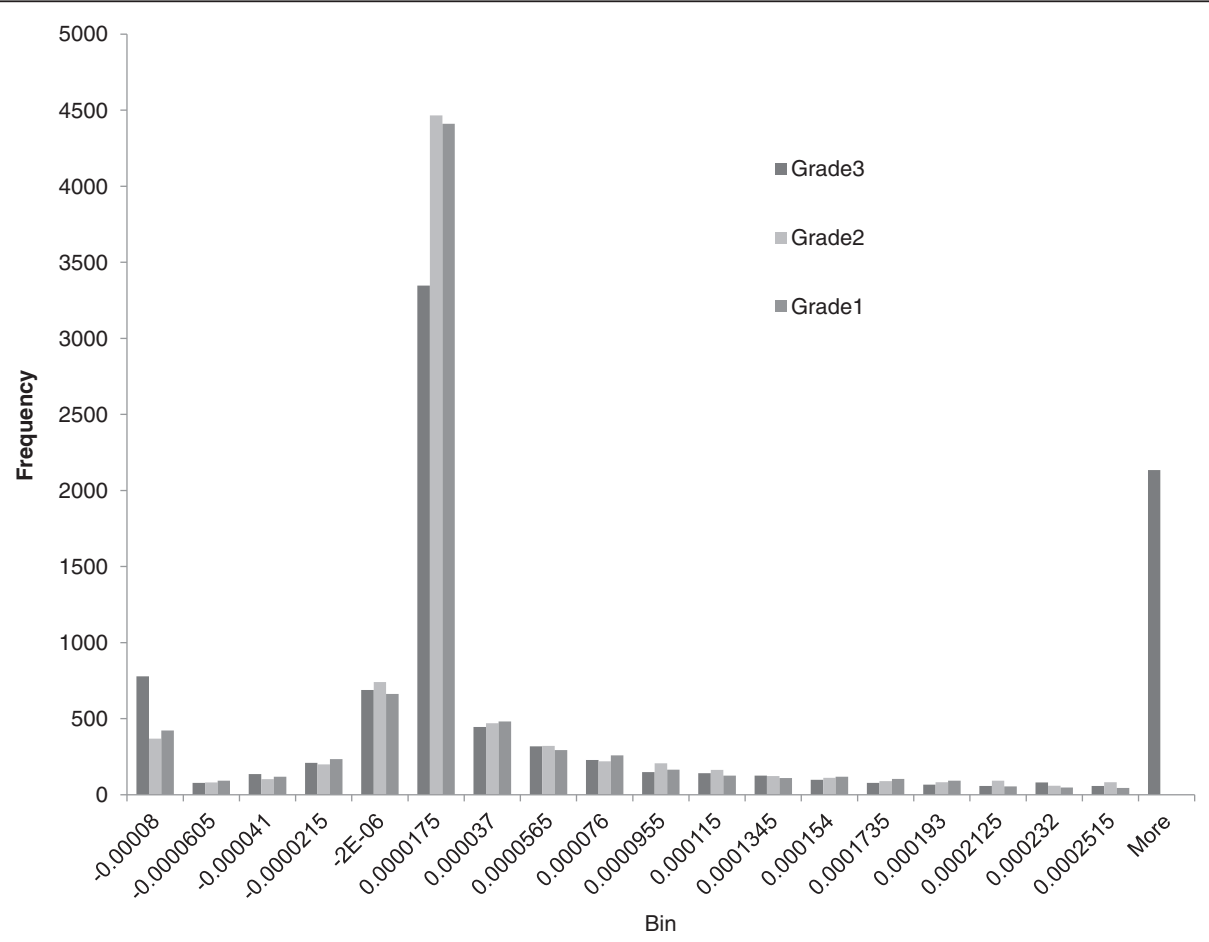

Figure 1 Comparison of $96 \mathrm{Ml}$ of grade1, grade2 and grade3 samples. 


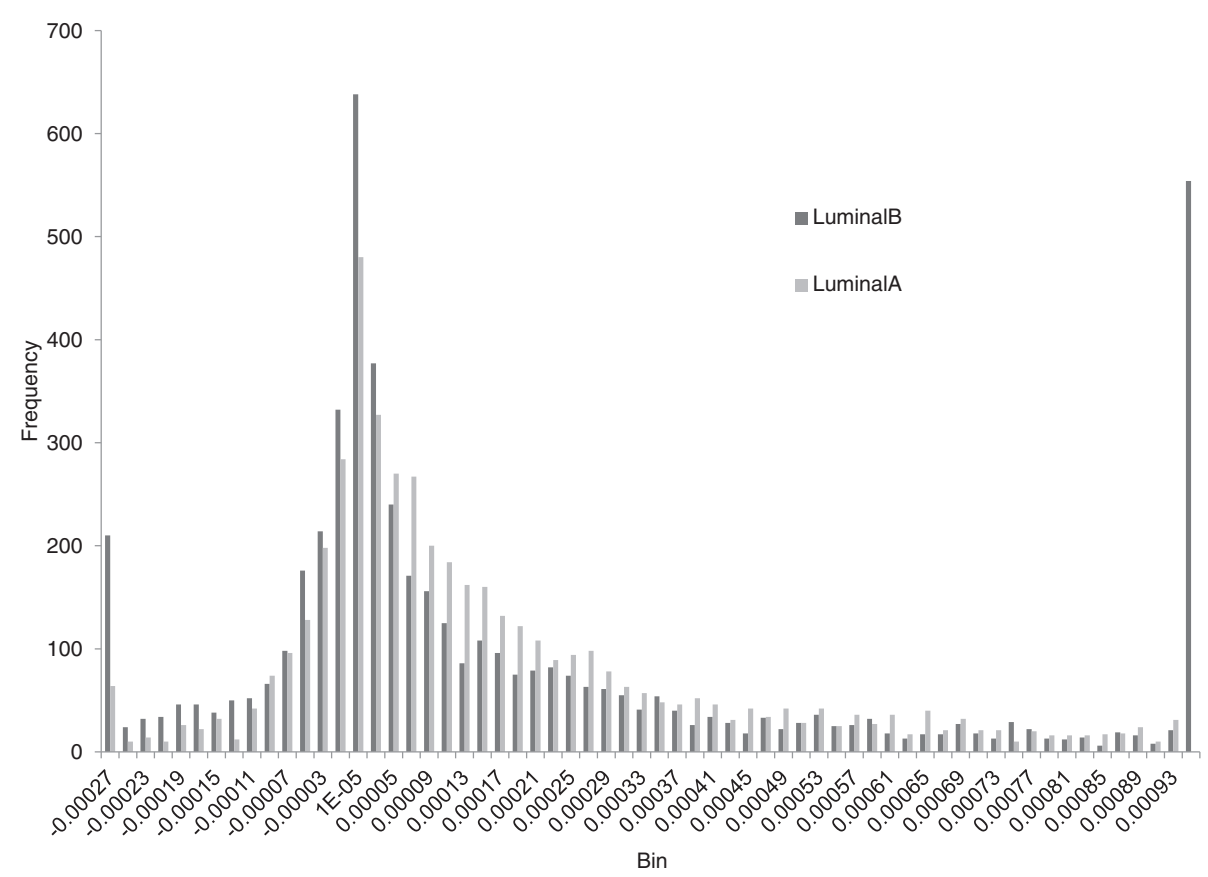

Figure 2 Comparison of $72 \mathrm{MI}$ of luminal A and luminal B samples.

forming interaction between biomarkers and drug agents to provide a personalized and synergistic therapy plan.

From the GEO datasets of cancers, we have discovered the genetic model of each cancer, called signature of that particular cancer. Among different cancers, their signatures (target networks) may be quite different which corresponds to different biomarkers in Additional file 1 GSE18655_96_ Biomarkers.xlsx, Additional file 2 GSE19536_72_Biomarkers.xlsx, Additional file 3 GSE19536_68_Biomarkers.xlsx, and Additional file 4 GSE21036_22_Biomakers.xlsx.. For these different signatures, we would discover various synergistic mechanisms which have exemplified in [24].

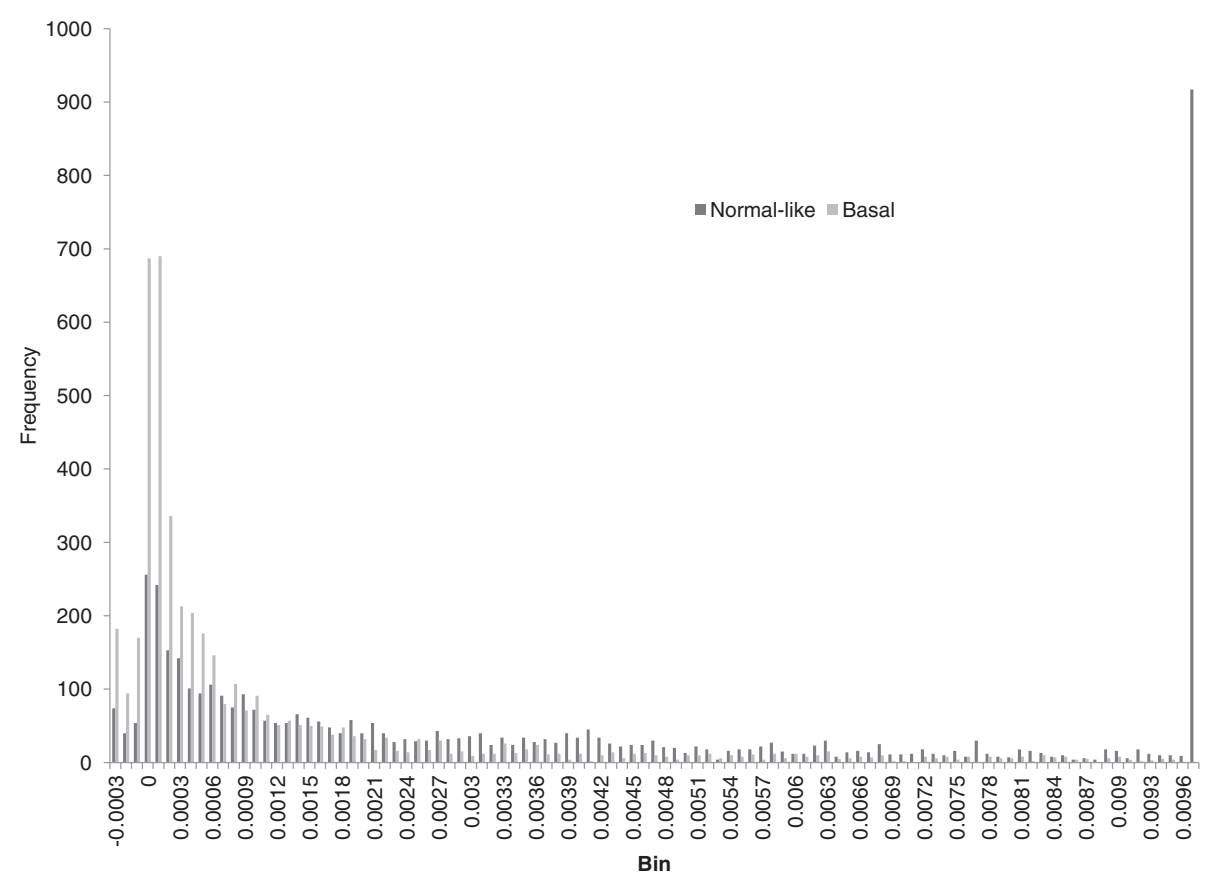

Figure 3 Comparison of $68 \mathrm{MI}$ of basal-like and normal-like samples. 


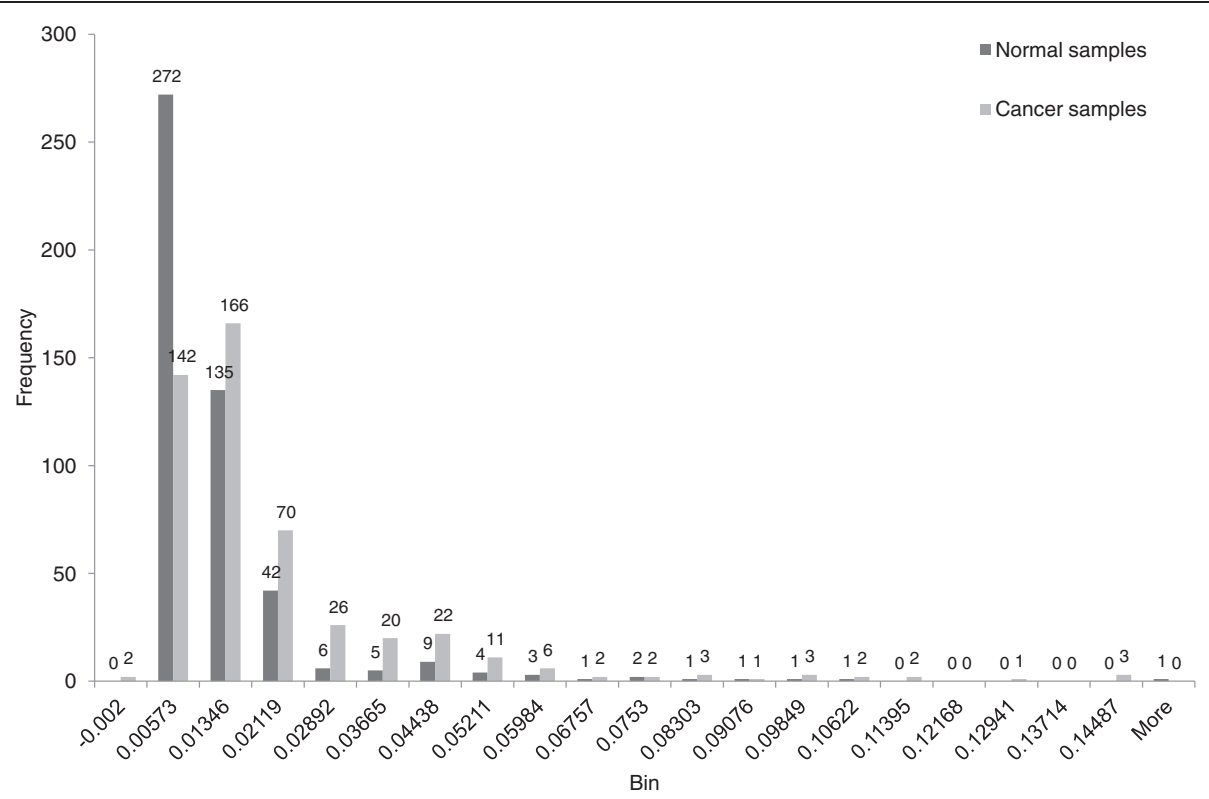

Figure 4 Comparison of $22 \mathrm{Ml}$ of prostate cancerous and normal samples.

Assume we would like to provide a synergistic therapy plan of a patient A. By collecting his/her bodily data such as saliva, blood samples, we first obtain the corresponding microarray dataset of patient A and apply it to the genetic model as shown in Figure 5.

A complete synergistic therapy should be able to select small subset of biomarkers and correlate them with drug agents in a multi-target multi-components network approach as shown in Figure 6. In Figure 6, a disease associates with several biomarkers such as RNAs, miRNAs or proteins denoted by $R_{1}, R_{2}, R_{3}, R_{4}$ and $R_{5}$ which are the regulators for operons $\mathrm{O}_{1}, \mathrm{O}_{2}$, and $\mathrm{O}_{3}$. An operon is a basic unit of DNAs and formed by a group of genes controlled by a gene regulator. These operons initiate molecular mechanisms as promoters. The gene regulators can enable organs to regulate other genes either by induction or repression. For each target biomarker, it may have a list of pharmacons used as enzyme inhibitors. Traditionally, pharmacons are referred to biological active substances which are not limited to drug agents only. For example, the herbal extractions whose ingredients have a promising anti-AD (Alzheimer's Disease) effect can be used as pharmacons [24]. Meanwhile, pharmacons denoted by $D_{1}, D_{2}$, and $D_{3}$, have effects for some target biomarkers. For example, $D_{1}$ affects target biomarker $R_{3}$, $\mathrm{D}_{2}$ affects target biomarker $\mathrm{R}_{5}$ and $\mathrm{D}_{3}$ affects biomarker $\mathrm{R}_{1}$. Compared with drug agent pair methodology [5], the proposed framework in Figure 6 represents a

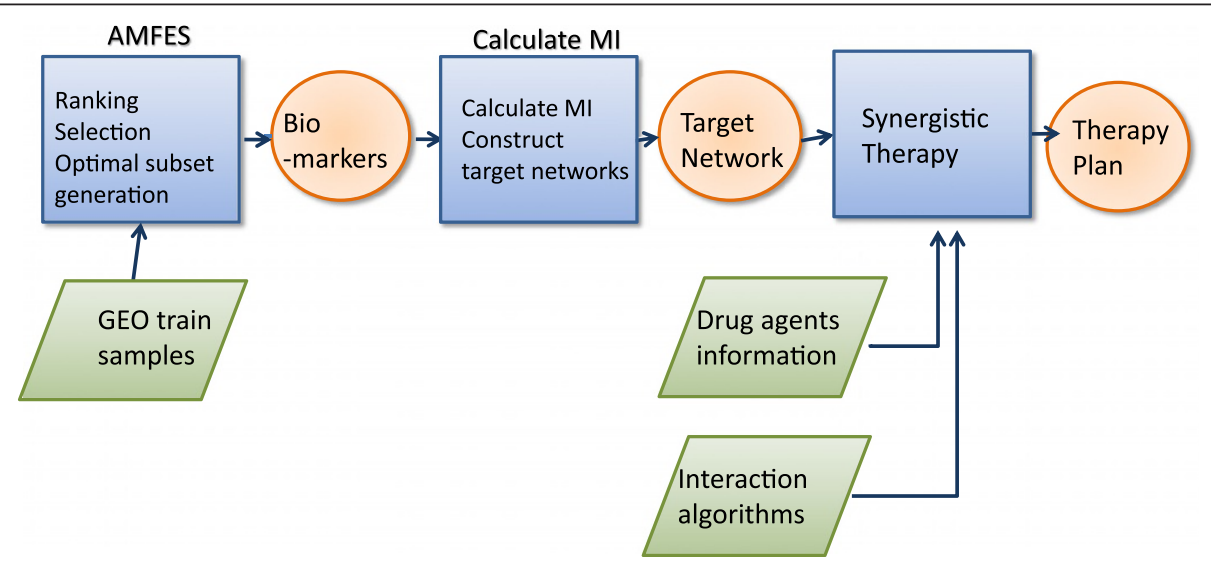

Figure 5 Diagram of detailed process of building the genetic model. 


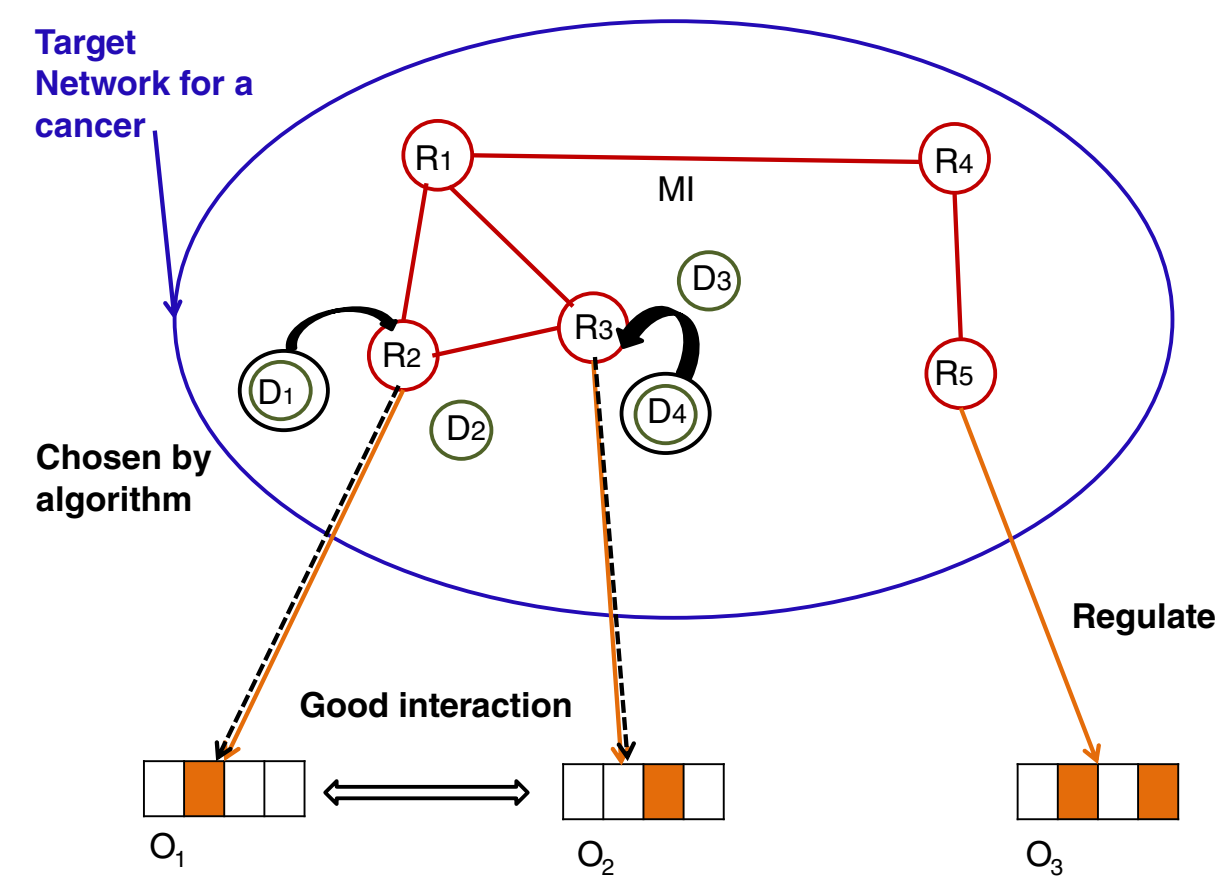

Figure 6 Relationships between biomarkers, pharmacons and operons where $R_{1}, R_{2}, R_{3}, R_{4}$ and $R_{5}$ denote 5 biomarkers. Among all the biomarkers, $R_{2}, R_{3}$ and $R_{5}$ are regulators.

more accurate interpretation of biomarkers with multicomponent drug agents.

\section{Discussion}

Among the MI values obtained, we see positive values and negative values. The positive value can represent the attractions among the biomarkers while the negative may represent the repulsion among the biomarkers, which matches the concept of Yin-Yang in TCM (Traditional Chinese Medicine). From these results, we observed that there is minimal difference of mutual information values between cancer stages. However, the difference of mean MI value of the prostate cancer versus normal cells is move obvious. The mean MI value of the last prostate cancer cell is approximately twice that of normal cells. This may be intriguing for medical people for further investigations.

\section{Conclusions}

We have presented a comprehensive approach to diagnosis and therapy of complex diseases, such as cancer. A complete procedure is proposed for clinical application to cancer patients. While the genetic model provides a standard framework to design synergistic therapy, the actual plan for individual patient is personalized and flexible. With careful monitoring, physicians may adaptively change or modify the therapy plan. Much further analysis of this framework in clinical settings should be experimented.

\section{Additional files}

Additional file 1: GSE18655_96_Biomarkers. An MS Office Excel file which contains a list of gene symbols of 96 biomarkers of GSE18655 samples.

Additional file 2: GSE19536_72_Biomarkers. An MS Office Excel file which contains a list of gene symbols of 72 biomarkers of GSE19536 luminal $A$ and luminal $B$ samples.

Additional file 3: GSE19536_68_Biomarkers. An MS Office Excel file which contains a list of gene symbols of 68 biomarkers of GSE19536 basal-like and normal-like samples.

Additional file 4: GSE21036_22_Biomarkers. An MS Office Excel file which contains a list of gene symbols of 22 biomarkers of GSE21036 samples.

Additional file 5: 18655 Grade1 MI. An MS Office Excel file which contains a matrix of the pairwise Ml values of 96 biomarkers of grade1 samples.

Additional file 6: 18655 Grade2 MI. An MS Office Excel file which contains a matrix of the pairwise MI values of 96 biomarkers of grade2 samples.

Additional file 7: 18655 Grade3 MI. An MS Office Excel file which contains a matrix of the pairwise Ml values of 96 biomarkers of grade 3 samples.

Additional file 8: 19536 Luminal-A MI. An MS Office Excel file which contains the pairwise MI values of 72 biomarkers of luminal A samples.

Additional file 9: 19536 Luminal-B MI. An MS Office Excel file which contains the pairwise $\mathrm{Ml}$ values of 72 biomarkers of luminal $\mathrm{B}$ samples. Additional file 10: 19536 Basal-Like MI. An MS Office Excel file which contains the pairwise Ml values of 68 biomarkers of Basal-like samples. 
Additional file 11: 19536 Normal-Like MI. An MS Office Excel file which contains the pairwise MI values of 68 biomarkers of Normal-like samples.

Additional file 12: 21036 Cancer MI. An MS Office Excel file which contains the pairwise $\mathrm{MI}$ values of 22 biomarkers of cancerous samples.

Additional file 13: 21036 Normal MI. An MS Office Excel file which contains the pairwise $\mathrm{Ml}$ values of 22 biomarkers of normal samples.

\section{Competing interests}

The authors declare that they have no competing interests.

\section{Author's contributions}

WH, CL: Implementation of project. FC, SC: Design the project. All authors read and approved the final manuscript.

\section{Acknowledgements}

We are grateful to the reviewers for their valuable comments and suggestions. We are also grateful to Dr. John Harris for his encouragements for this research. We are also thankful for Dr. Lung-Ji Chang for his discussion and encouragements.

\section{Author details}

${ }^{1}$ System Biology Lab, University of Florida, Florida, USA. ${ }^{2}$ Department of Electrical and Computer Engineering, University of Florida, Florida, USA. ${ }^{3}$ Department of Computer and Information Science and Engineering, University of Florida, Florida, USA. ${ }^{4}$ Institute of Information Science, Academia Sinica, Taipei, Taiwan.

Received: 10 July 2012 Accepted: 20 September 2012

Published: 2 October 2012

\section{References}

1. Zimmermann GR, Lehar J, Keith CT: Multi-target therapeutics: when the whole is greater than the sum of the parts. Drug discovery today 2007, 12 (1-2):34-42.

2. Keith CT, Borisy AA, Stockwell BR: Multicomponent therapeutics for networked systems. Nat Rev Drug Discov 2005, 4(1):71-78.

3. Dancey JE, Chen HX: Strategies for optimizing combinations of molecularly targeted anticancer agents. Nature reviewsDrug discovery 2006, 5(8):649-659.

4. Csermely $P$, Agoston $V$, Pongor S: The efficiency of multi-target drugs: the network approach might help drug design. Trends Pharmacol Sci 2005, 26 (4):178-182.

5. Li S, Zhang B, Zhang N: Network target for screening synergistic drug combinations with application to traditional Chinese medicine. BMC Syst Biol 2011, 5(Suppl 1):S10. Journal Article.

6. Hsu W-C, Liu C-C, Chang F, Chen S-S: Feature Selection for Microarray Data Analysis: GEO \& AMFES. Gainesville, Florida: Technical Report; 2012.

7. Guyon I, Weston J, Barnhill S, Vapnik V: Gene Selection for Cance Classification using Support Vector Machines. Mach Learn 2002, 46(1-3):389-422

8. Rakotomamonjy A: Variable selection using svm based criteria. J Mach Learn Res 2003, 3:1357-1370.

9. Bi J, Bennett K, Embrechts M, Breneman C, Song M: Dimensionality reduction via sparse support vector machines. J Mach Learn Res 2003 3:1229-1243.

10. Stoppiglia H, Dreyfus $G$, Dubois $R$, Oussar $Y$ : Ranking a random feature for variable and feature selection. JMachLearnRes 2008, 3(Journal Article):1399-1414.

11. Tuv E, Borisov A, Torkkola K: Feature Selection Using Ensemble Based Ranking Against Artificial Contrasts. In Neural Networks, 2006 IJCNN '06 International Joint Conference on: 0-0 0: 2006:2181-2186.

12. Shannon CE: A mathematical theory of communication. SIGMOBILE Mob Comput Commun Rev 2001, 5(1):3-55.

13. Qiu P, Gentles AJ, Plevritis SK: Fast calculation of pairwise mutual information for gene regulatory network reconstruction. Comp Methods and Programs in Biomed 2009, 94(2):177-180.

14. Beirlant J, Dudewicz EJ, ouml LG,r, Meulen ECVD: Nonparametric entropy estimation: An overview. Int J Math Stat Sci 1997, 6(1):17-39.
15. Margolin A, Nemenman I, Basso K, Wiggins C, Stolovitzky G, Favera R, Califano A: ARACNE: An Algorithm for the Reconstruction of Gene Regulatory Networks in a Mammalian Cellular Context. BMC Bioinforma 2006, 7(Suppl 1):S7.

16. Michael EW, Monica SL: A data locality optimizing algorithm.; 1991.

17. Fitzgerald JB, Schoeberl B, Nielsen UB, Sorger PK: Systems biology and combination therapy in the quest for clinical efficacy. Nat Chem Biol 2006, 2(9):458-466

18. Page L, Brin S, Motwani R, Winograd T: The PageRank Citation Ranking: Bringing Order to the Web. In Stanford InfoLab. 1999.

19. van Driel MA, Bruggeman J, Vriend G, Brunner HG, Leunissen JA: A textmining analysis of the human phenome. Eur J Human Genet : EJHG 2006 14(5):535-542.

20. Sobin LH, Wittekind C: TNM: classification of malignant tumours. New York: Wiley-Liss; 2002

21. Barwick BG, Abramovitz M, Kodani M, Moreno CS, Nam R, Tang W, Bouzyk $M$, Seth A, Leyland-Jones B: Prostate cancer genes associated with TMPRSS2-ERG gene fusion and prognostic of biochemical recurrence in multiple cohorts. Br J Cancer 2010, 102(3):570-576.

22. Enerly E, Steinfeld I, Kleivi K, Leivonen SK, Aure MR, Russnes HG, Ronneberg

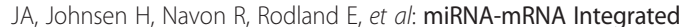
Analysis Reveals Roles for miRNAs in Primary Breast Tumors. PLoS One 2011, 6(2):e16915.

23. Taylor BS, Schultz N, Hieronymus H, Gopalan A, Xiao Y, Carver BS, Arora VK, Kaushik $P$, Cerami E, Reva B, et al: Integrative genomic profiling of human prostate cancer. Cancer cell 2010, 18(1):11-22.

24. Sun Y, Zhu R, Ye H, Tang K, Zhao J, Chen Y, Liu Q, Cao Z: Towards a bioinformatics analysis of anti-Alzheimer's herbal medicines from a target network perspective. In Briefings in bioinformatics. 2012.

doi:10.1186/2043-9113-2-16

Cite this article as: Hsu et al:: Cancer classification: Mutual information target network and strategies of therapy. Journal of Clinical Bioinformatics 2012 2:16.

\section{Submit your next manuscript to BioMed Central and take full advantage of:}

- Convenient online submission

- Thorough peer review

- No space constraints or color figure charges

- Immediate publication on acceptance

- Inclusion in PubMed, CAS, Scopus and Google Scholar

- Research which is freely available for redistribution 